\title{
Sustained visual attention in deaf and hearing adults
}

\author{
MARY LYNNE DITTMAR, DANIEL B. BERCH, and JOEL S. WARM \\ University of Cincinnati, Cincinnati, Ohio 45221
}

\begin{abstract}
Benedetti and Loeb (1972) have established that loss of vision (blindness) can be associated with enhancement in the quality of auditory vigilance. The present study demonstrates that loss of hearing (deafness) can result in an analogous enhancement with regard to visual vigilance. Legally deaf and normal-hearing adults monitored a visual display continuously for $45 \mathrm{~min}$. Critical signals for detection were occasional increments in the horizontal movements of a bar of light. The deaf observers detected significantly more signals with no higher false alarm rates than the normals. The superiority of the deaf is attributed to the transfer of practiced variables related to more efficient utilization of visual information.
\end{abstract}

Sensory factors are of considerable importance in the performance of sustained attention or vigilance tasks. For example, the overall quality of sustained attention is greater when stimulation is in the auditory as compared with the visual mode, and in some cases, acoustic tasks have been more resistant than visual tasks to the temporal decline in performance that typically occurs in vigilance situations (cf. Warm, 1977). In addition, similar variations in task parameters are not necessarily accompanied by parallel changes in auditory and visual performances (Loeb \& Binford, 1968).

As a rule, knowledge of sensory factors in vigilance has come from experiments using observers who were free of sensory impairments. However, insight into the role played by sensory factors in monitoring activities might also be gained by considering the quality of sustained attention in sensory-handicapped individuals. One might wonder if the presence of such a handicap in a given modality (e.g., vision) has any bearing on an individual's ability to sustain attention to stimulation in a nonimpaired modality (e.g., audition). In addition to its implications for an understanding of sensory factors in vigilance, a question of this sort also has meaning on an organismic level, since individual differ-

This project was supported by Grant MCT-000-912-16-0, awarded to the Bureau of Community Health Services, by the Health Services Administration, Public Health Service, DHEW, and by Grant 59-P-25297/5-08, awarded by Region V, Social and Rehabilitation Service, DHEW. The authors are grateful to the following individuals for their assistance in securing deaf participants: William DeMeo (St. Rita School for the Deaf), Daniel Langholtz (Cincinnati Speech and Hearing Center), Doris Mangold, Connie Ritze, and Rosie Schwartz. Thanks are also extended to William DeMeo, John Bowers, Clare Kibler, and Jacqueline Williamson for giving instructions to the deaf in ASL, and to Ruth Vernier for her assistance in testing subjects. Daniel B. Berch is also affiliated with the Department of Psychology, Cincinnati Center for Developmental Disorders, Elland and Bethesda Avenues, Cincinnati, Ohio 45229. Requests for reprints should be sent to him at that address. ences are a critical factor in the quality of vigilance performance (Berch \& Kanter, in press; Davies \& Parasuraman, 1981).

The initial experimental effort to probe the quality of sustained attention in sensory-handicapped individuals was made by Benedetti and Loeb (1972). These investigators compared the performance of blind and sighted adults on an auditory vigilance task and found that loss of vision did indeed have an impact on the ability of blind listeners to sustain auditory attention. The blind were superior to the sighted in terms of both the number of signals detected and effective sensitivity ( $\left.\mathrm{d}^{\prime}\right)$.

The work of Benedetti and Loeb (1972) has established that loss of vision can be associated with enhancement in the quality of sustained auditory attention. To date, no attempt has been made to continue this line of investigation and determine if an analogous enhancement occurs in visual vigilance when the performances of deaf and normal-hearing individuals are compared. The absence of such an attempt may reflect the assumption that superiority in visual vigilance would be an anticipated consequence of deafness and, therefore, that its demonstration would be experimentally uninteresting. Nevertheless, a consideration of some cognitive and perceptual correlates of deafness suggests that finding enhanced visual vigilance in the deaf is not at all certain.

Unlike the blind, who frequently exceed the sighted on many tasks requiring acoustic functions (Benedetti $\&$ Loeb, 1972; Warren, 1978), the deaf have generally been found to perform either as well as or more poorly than normal-hearing controls on a wide spectrum of tasks involving visual functions. These include signal detection (Bross, 1979), temporal acuity (critical flicker fusion; Bross \& Sauerwein, 1980), and word recognition tasks (Doehring \& Rosenstein, 1960), as well as visual short-term memory (Carey \& Blake, 1974), and visual search tasks (Henderson \& Henderson, 1973). Moreover, few deaf individuals attain levels of school achievement or acquire reading skills commensurate 
with those of their normal-hearing peers (Myklebust, 1960), and evidence is accumulating to indicate that both school achievement and reading ability are positively related to vigilance performance (Berch \& Kanter, in press). Taken together, these somewhat scant, but nevertheless compelling, findings imply that enhanced visual vigilance may not necessarily be a correlate of deafness. Rather, the deaf may be poorer than normalhearing individuals in performing a visual vigilance task. Clearly, the matter warrants experimental scrutiny. Such scrutiny was the purpose of the present investigation.

\section{METHOD}

\section{Subjects}

Twenty deaf and 50 normal-hearing individuals served as subjects. The deaf were recruited from a school for the deaf and from various clubs and organizations in the Cincinnati metropolitan area. To encourage participation, an award of $\$ 20$ was offered for the best overall performance by a deaf individual on the vigilance task. The mean age of the deaf participants was 21 years (range $=18-24$ years). They all had normal or correctedto-normal vision and were free of any gross motor impairments. Each deaf participant had been classified as legally deaf, and the affliction was either congenital or had appeared before the age of 2 years. The education level of the deaf subjects ranged from eighth grade through graduate training. On average, the deaf participants had completed 13 years of formal schooling.

The normal-hearing subjects were students from the University of Cincinnati who participated in order to fulfill a course requirement. Two groups of 25 normal-hearing subjects were formed. The subjects in one of these groups, the hearing/no-prize condition, received only course credit for their participation. The subjects in the other group, the hearing/prize condition, received course credit for participating and also had the opportunity to compete for a $\$ 20$ prize to be awarded to the student with the best overall performance on the vigilance task. The latter condition was included to control for the possible effects of extrinsic reward introduced by offering the deaf subjects a financial prize as an inducement to serve in the experiment. In both the hearing/no-prize and hearing/prize groups, the mean age wàs 20 years (the age ranges were 18-24 and 18-29 years, respectively). All of the normal-hearing subjects had normal or corrected-to-normal vision. None of the participants in the study had prior experience with vigilance experiments. ${ }^{1}$

\section{Apparatus and Procedure}

The subjects monitored the apparent movement of an $18 \mathrm{x}$ $2 \mathrm{~mm}$ bar of red light that traveled along a horizontal vector within an $18 \times 32 \mathrm{~mm}$ window. The window was centered within a $555 \times 375 \mathrm{~mm}$ flat-black panel. Apparent movement of the light bar was produced by successively illuminating small, and appropriately placed, Plexiglas diffusing screens. A neutral event, to which no overt response was required, was a pair of movements in which the bar moved $24 \mathrm{~mm}$ to the right (movement time, $.60 \mathrm{sec}$ ), snapped back to its start position (where it remained for $.80 \mathrm{sec}$ ), again moved $24 \mathrm{~mm}$ to the right (movement time, $.60 \mathrm{sec}$ ), and then snapped back to its start position, where it remained for the ensuing interevent interval. The light bar executed 21 paired movements per minute. This event rate was achieved by setting the interevent intervals at $.90 \mathrm{sec}$.

Critical signals for detection were increments of $2 \mathrm{~mm}$ (24-26 mm) in the second deflection within a pair of movements. This value represented an increment of $8.3 \%$ of the base movement. An $18 \times .5 \mathrm{~mm}$ white sight bar was positioned in vertical alignment $24 \mathrm{~mm}$ from the left end of the window in the display. The depth separation between the sight bar and the display was $6 \mathrm{~mm}$. The sight bar served to mark the end of a nonsignal deflection. With a two-alternative temporal forcedchoice procedure, critical deflections were essentially always detected by alerted subjects. The observers indicated their detection of a critical signal by pressing a hand-held microswitch. The moving bar display used in this study was adopted from Jerison and Pickett (1964). It is designed to minimize memory demands as to the nature of a critical signal by having all the information needed to make a paired comparison judgment available within an event.

All subjects participated in a session consisting of a 5-min pretest phase, a 45-min vigil divided into three continuous 15-min periods, and a 5-min posttest phase. The subjects rested for $3 \mathrm{~min}$ following the pretest and vigilance phases of the experimental session. Five critical signals were presented during the pretest and posttest phases. The intersignal intervals in these phases ranged from 45 to $75 \mathrm{sec}$, with a mean of $60 \mathrm{sec}$. Five critical signals were also presented per period during the main watch. The intersignal intervals during each period of the main watch were $30,105,180,255$, and $330 \mathrm{sec}$ (mean $=180 \mathrm{sec})$. Intersignal intervals were randomly ordered for each subject during the pretest and posttest portions of the experimental session and for each period during the main watch.

Neutral events and the occurrence of critical signals were controlled by solid state programming equipment and a Gerbrands punched-tape timer. The subject's responses were recorded on two ITT electronic counters. Responses occurring within $2.5 \mathrm{sec}$ after the onset of critical signals were recorded automatically as correct detections; all others were considered as errors of commission or false alarms. The $2.5 \mathrm{sec}$ cutoff value was based upon previous work with this display (Warm, Wait, \& Loeb, 1976), which indicated that if subjects were going to respond to a critical signal, they would do so within this period of time.

Subjects were tested individually in a $1.8 \times 1.9 \times 2.0 \mathrm{~m}$ Industrial Acoustics sound chamber. The display to be monitored was mounted on a stand slightly below eye level. The subject viewed the display without restraint from a distance of approximately $520 \mathrm{~mm}$. Ambient illumination was provided by a 40-W bulb mounted in a parabolic reflector aimed at a wall of the chamber. Control equipment and the experimenter were located outside the chamber. An intercom permitted voice communication, as well as acoustic surveillance of the subject's activities during the experimental session. The subjects were informed that critical signals would occur much less frequently during the vigil than during the pretest. All participants surrendered their watches prior to the start of the session, and they had no knowledge as to the length of the session other than it would not exceed $1.5 \mathrm{~h}$. Deaf subjects received their instructions through American Sign Language (ASL).

\section{RESULTS}

Mean percentages of correct detections for the deaf and the two normal-hearing groups are plotted as a function of periods of watch in Figure 1. Data for the pretest and posttest phases of the experiment are also plotted in the figure.

It is evident in the figure that the general trend of performance was similar for all three groups of subjects. For each group, the trend consisted of a high level of detections during the pretest phase, a monotonic decline in detections over time during the main watch, and a rebound in the rate of detections during the posttest phase to a level that approximated that of the pretest. 


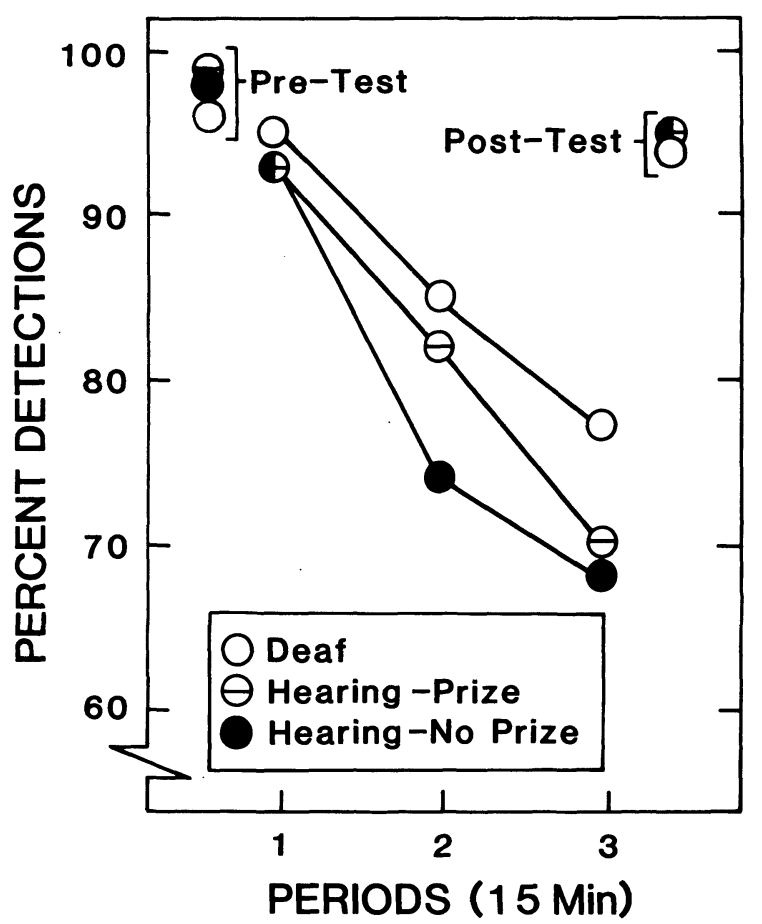

Figure 1. Percentage of correct detections for the deaf and the two normal-hearing groups during the pretest, posttest, and vigilance phases of the experiment. Vigilance data are plotted as a function of periods of watch.

It is also evident in Figure 1 that there was a relatively small but consistent difference in the detection scores of the three groups of subjects during the vigilance portion of the experiment. At each period of watch, the scores for the deaf group were above those for either of the normal-hearing groups.

The data of the pretest and posttest phases were converted to arcsins and subjected to separate one-way analyses of variance. These analyses revealed no significant differences in the detection scores of the three groups of subjects during the initial and final phases of the study $(p>.05)$. The data from the vigilance portion of the experiment were also converted to arcsins and entered into a two-factor analysis of variance in which an unweighted-means solution was used to accommodate unequal sample sizes (Kirk, 1968). This analysis revealed that there was a significant difference among the groups in overall detections during the vigil $[F(2,67)=4.16$, $\mathrm{p}<.025]$ and that performance efficiency during the vigil declined significantly over time $[\mathrm{F}(2,134)=19.14$, $\mathrm{p}<.001]$. The Groups by Periods interaction in this analysis lacked significance $(F<1)$. Thus, the rate of the temporal decline in detections was similar for the three groups of subjects. Newman-Keuls tests were employed to probe the differences in overall detection scores among the groups. The harmonic mean of the three sample sizes was used in computing the standard error component in these tests, and an alpha level of .05 was set for all comparisons. The tests revealed that the deaf group exhibited a significantly higher overall detection rate than both the hearing/prize and hearing/ no-prize groups and that the two normal-hearing groups did not differ significantly from each other.

An examination of the data for errors of commission showed that such errors were rare. In each phase of the experiment, the mean false alarm rate for each of the three groups of subjects was less than $.5 \%$.

\section{DISCUSSION}

The major finding of this experiment is that the deaf exhibited higher detection scores throughout the vigilance portion of the study than did either of the normal-hearing groups. While the margin of deaf superiority was relatively small, it was statistically significant and it cannot be attributed to artifacts such as a lowered level of general responsiveness on the part of the normals or capricious responding on the part of the deaf. The first artifact is ruled out by the finding of high pretest detection scores for all groups coupled with a rebound in detections for all groups during the posttest to approximately the pretest level. The second artifact is counterindicated by the rarity of false alarms in the data of all three groups. Clearly, the greater overall detection rate among the deaf was not accompanied by a high rate of commission errors that would have reflected capricious responding or an exceptionally lenient response criterion. Finally, the superior overall performance of the deaf in relation to the hearing/prize group indicates that a greater level of extrinsic motivation was not the basis for the superior performance of the deaf observers. All in all, it appears that the present results point to a hearing-loss analog to Benedetti and Loeb's (1972) earlier finding in regard to loss of vision. Just as blind listeners show enhancement in the quality of sustained auditory attention, deaf observers show enhancement in the quality of sustained visual attention.

Several possibilities might be offered to account for the superior performance of the deaf in this investigation. One of these stems from the use of a sound-attenuation booth to house the subjects. While such housing is common in vigilance experiments, it does present normal-hearing subjects with an element of acoustic impoverishment to which they are not normally exposed. In contrast, deaf subjects face such impoverishment continuously and therefore may have found the vigilance situation to be less unsettling than did the normals. The potential validity of this sort of explanation is diminished, however, by the fact that deaf individuals tend to exhibit high degrees of suspicion (Ramsdell, 1970), a tendency that might be particularly likely to manifest itself in unfamiliar and "closed-in" environments. Such a reaction should have offset any benefit accruing to the deaf as a result of housing normal-hearing subjects in the sound-attenuation booth. Indeed, several of the deaf participants voiced discomfort at being left alone in the booth and had to be reassured. No such reaction was noted among the normal-hearing individuals.

It might be tempting to account for the heightened performance of the deaf in this study on the basis of the classical notion of "sensory compensation" (cf. Hayes, 1933), that is, a heightened physiological sensitivity to visual stimuli. Benedetti and Loeb (1972) also considered this possibility in accounting for their finding of superior auditory vigilance in the blind. They argued, however, that since they could find no significant differences between their blind and sighted subjects on several auditory signal detection and threshold tasks, their vigilance findings were unlikely to be rooted in a general increase in physiological sensitivity among the blind. While the present 
experiment did not compare deaf and normal-hearing observers on visual signal detection and threshold tasks, there is reason to believe that "sensory compensation" is also inadequate to account for the present results. If deaf individuals do indeed benefit from a general increase in physiological sensitivity to visual stimuli, one would expect to find deaf superiority on a variety of visual tasks. As noted previously, however, this is generally not the case.

As an alternative to "sensory compensation," Benedetti and Loeb (1972) offered a perceptual learning notion to account for the superior performance of the blind subjects in their experiment. They pointed out that because blind persons have lost a rich source of visual information, they have learned to rely more on hearing than do the sighted, particularly in circumstances under which observers are uncertain as to when the critical events to be detected will occur. Temporal uncertainty was an important element in Benedetti and Loeb's study as it is in most vigilance experiments (cf. Warm, 1977). Thus, Benedetti and Loeb suggested that the superior performance of the blind listeners in their investigation was due to the transfer to the vigilance situation of practiced skills in listening for and discriminating among critical auditory events in a temporally uncertain context.

In similar fashion, the transfer of compensatory perceptual skills to the vigilance task may also underlie the superior performance of the deaf subjects in this investigation. It is possible that because they do not have a rich source of auxiliary acoustic information available to them, deaf adults have learned to use their visual systems more efficiently than normals in coping with the temporal uncertainty associated with the appearance of critical stimuli in their field of view. Such learning could have been of benefit to the deaf in the vigilance portion of the present study, since the temporal separations between critical signal presentations were quite irregular, ranging from intervals as short as $30 \mathrm{sec}$ to those as long as $330 \mathrm{sec}$.

Along these lines, it is worth noting that in the normal perceptual world, the detection of stimuli that appear in spatially uncertain locations in the field of view is frequently aided by redundant auditory cues. Since the deaf do not have access to such cues, they may have developed more proficiency than normals in scanning the visual field for spatially uncertain events. Consequently, the deaf may also be more able than normals to cope with another source of uncertainty known to degrade vigilance performance: spatial uncertainty, or uncertainty as to where critical signals will appear (cf. Warm, 1977). Such uncertainty was not incorporated into the design of the present study. Therefore, it is conceivable that the results of this investigation may actually underestimate the degree to which deaf observers exceed normals in the overall quality of sustained visual attention. If a vigilance task were used in which both spatial and temporal uncertainty were involved, the gap in performance efficiency between deaf and normal-hearing observers might widen still further in favor of the deaf.

\section{REFERENCES}

Benedetti, L. H., \& Loeb, M. A comparison of auditory monitoring performance in blind subjects with that of sighted sub- jects in light and dark. Perception \& Psychophysics, 1972, 11, 10-16.

BerCh, D. B., \& KANTER, D. R. Individual differences. In J. S. Warm (Ed.), Sustained attention in human performance. London: Wiley, in press.

Bross, M. Residual sensory capacities of the deaf: A signal detection analysis of a visual discrimination task. Perceptual and Motor Skills, 1979, 48, 187-194.

Bross, M., \& SAUERwein, H. Signal detection analysis of visual flicker in deaf and hearing individuals. Perceptual and Motor Skills, 1980, 51, 839-843.

CAREY, P., \& Blake, J. Visual short-term memory in the hearing and the deaf. Canadian Journal of Psychology, 1974, 28, 1-14.

Davies, D. R., \& Parasuraman, R. The psychology of vigilance. London: Academic Press, 1981.

Doenring, D. G., \& Rosenstein, J. Visual word recognition by deaf and hearing children. Journal of Speech and Hearing Research, 1960, 3, 320-326.

HAYES, S. P. New experimental data on the old problem of sensory compensation. Teachers Forum, 1933, 5, 22-26.

Henderson, S. E., \& Henderson, L. Levels of visualinformation processing in deaf and hearing children. American Journal of Psychology, 1973, 86, 507-521.

Jerison, H. J., \& Pickett, R. M. Vigilance: The importance of the elicited observing rate. Science, 1964, 143, 970-971.

KIrK, R. E. Experimental design: Procedures for the behavioral sciences. Belmont, Calif: Brooks/Cole, 1968.

LOEB, M., \& Binford, J. R. Variation in performance on auditory and visual monitoring tasks as a funcion of signal and stimulus frequencies. Perception \& Psychophysics, 1968, 4, 361367.

Myklebust, H. R. The psychology of deafness. New York: Grune \& Stratton, 1960.

RAMSDELL, D. A. The psychology of the hard-of-hearing and the deafened adult. In H. Davis \& S. R. Silverman (Eds.), Hearing and deafness (3rd ed.). New York: Holt, Rinehart \& Winston, 1970.

WARM, J. S. Psychological processes in sustained attention. In R. R. Mackie (Ed.), Vigilance: Theory, operational performance, and physiological correlates. New York: Plenum, 1977.

WARM, J. S., Wait, R. G., \& Loeb, M. Head restraint enhances visual monitoring performance. Perception \& Psychophysics, 1976, 20, 299-304.

Warren, D. H. Perception by the blind. In E. C. Carterette \& M. P. Friedman (Eds.), Handbook of perception: Perceptual ecology (Vol. 10). New York: Academic Press, 1978.

\section{NOTE}

1. At the outset of this investigation, it was intended that there would be 25 subjects in each of the three experimental groups. However, we were able to secure only 20 legally deaf individuals who met the criteria of early-onset deafness, freedom from visual or motor impairments, and at least an eighth-grade education.

(Received for publication April 30, 1982.) 